\title{
Gene expression profiling of light-induced retinal degeneration in phototransduction gene knockout mice
}

\author{
Jayalakshmi Krishnan ${ }^{1 *}$, Jiayan Chen $^{2 *}$, \\ Kum-Joo Shin ${ }^{3 *}$, Jong-lk Hwang ${ }^{4}$, Sang-Uk Han ${ }^{1,5}$, \\ Gwang Lee ${ }^{1,6,8}$ and Sangdun Choi ${ }^{1,7,8}$ \\ ${ }^{1}$ Department of Molecular Science and Technology \\ Ajou University \\ Suwon 443-749, Korea \\ ${ }^{2}$ Zilkha Neurogenetic Institute \\ University of Southern California \\ Los Angeles, CA 90033, USA \\ ${ }^{3}$ Division of Biology \\ California Institute of Technology \\ Pasadena, CA 91125, USA \\ ${ }^{4}$ Graduate School of Medicine \\ Korea University \\ Seoul 136-705, Korea \\ ${ }^{5}$ Department of Surgery \\ ${ }^{6}$ Brain Disease Research Center \\ Ajou University School of Medicine \\ ${ }^{7}$ Department of Biological Sciences \\ College of Natural Science, Ajou University \\ Suwon 443-749, Korea \\ ${ }^{8}$ Corresponding authors: Tel, 82-31-219-2600; Fax, 82-31-219-1615; \\ E-mail, sangdunchoi@ajou.ac.kr (S. Choi) \\ Tel, 82-31-219-4554; Fax, 82-31-216-6381; \\ E-mail, glee@ @ajou.ac.kr (G. Lee) \\ *These authors contributed equally to this work. \\ DOI 10.3858/emm.2008.40.5.495
}

Accepted 15 May 2008

Abbreviations: GPCR, G protein coupled receptor; Rhok, Rhodopsin kinase; Sag, $S$ antigen (Arrestin)

\begin{abstract}
Exposure to light can induce photoreceptor cell death and exacerbate retinal degeneration. In this study, mice with genetic knockout of several genes, including rhodopsin kinase ( $\left.\mathrm{Rhok}^{-/}\right)$, arrestin ( $\left.\mathrm{Sag}^{-/}\right)$, transducin (Gnat $\left.1^{-1}\right)$, c-Fos (c-Fos $\left.{ }^{-1}\right)$ and arrestin/transducin $\left(\mathrm{Sag}^{-/ /} / \mathrm{Gnat1}^{-1}\right)$, were examined. We measured the expression levels of thousands of genes in order to investigate their roles in phototransduction signaling in light-induced retinal degeneration using DNA microarray technology and then further explored the gene network using pathway analysis tools. Several cas-
\end{abstract}

cades of gene components were induced or inhibited as a result of corresponding gene knockout under specific light conditions. Transducin deletion blocked the apoptotic signaling induced by exposure to low light conditions, and it did not require c-Fos/AP-1. Deletion of $c$-Fos blocked the apoptotic signaling induced by exposure to high intensity light. In the present study, we identified many gene transcripts that are essential for the initiation of light-induced rod degeneration and proposed several important networks that are involved in pro- and anti-apoptotic signaling. We also demonstrated the different cascades of gene components that participate in apoptotic signaling under specific light conditions.

Keywords: arrestin; gene expression profiling; oligonucleotide array sequence analysis; proto-oncogene proteins c-fos; retinal degeneration; transducin

\section{Introduction}

Apoptosis is the final common pathway in many cases of retinal degeneration or retinal disorders, such as retinitis pigmentosa (RP), and these disorders might be ameliorated by interfering with this process (Chang et al., 1993; Portera-Cailliau et al., 1994). Retinal degeneration and its associated signaling networks have attracted much research interest for many years. Several reports have shown that light induces apoptosis in the retina and that components of AP-1 are involved in this process (Hafezi et al., 1997; Hao et al., 2002). However, the signaling networks that initiate the retinal degeneration cascade are not fully understood. Transducin is a heterotrimeric $G$ protein expressed in the rods and cones of the vertebrate retina, and knockout studies of transducin (Gnat $1^{-/}$) showed no alteration in retinal morphology (Makino et al., 2003). However, there was an abrogation of signaling from light-activated rhodopsin (Calvert et al., 2000). Light activates rhodopsin, which is phosphorylated by rhodopsin kinase and the signal is terminated by arrestin, which binds specifically with phosphorylated rhodopsin (Dolph, 2002; Miller and Lefkowitz, 2001).

Rhodopsin signaling is prolonged in transgenic mice with null mutations in the genes encoding rhodopsin kinase $\left(\right.$ Rhok $\left.^{-/}\right)$(Chen et al., 1999a), 
arrestin $\left(\mathrm{Sag}^{-/}\right)$(Chen et al., 1999b), or rhodopsin kinase/arrestin ( Rhok $^{-/} / \mathrm{Sag}^{-/}$) (Choi et al., 2001). In addition, c-Fos is known to be a mediator of apoptosis, but its precise role in light-induced apoptosis is unclear. Therefore, we also used $c-\mathrm{Fos}^{-\alpha}$ mice to identify the genes affected. We screened the gene expression pattern in the retina of mice with knockout of key genes involved in phototransduction. Knockout strategies would provide a great deal of information regarding the functions of genes in mammals. Understanding the mechanism of signaling networks in visual systems will be greatly facilitated by the characterization of transcriptional regulation in genetic knockouts.

There are two types of light-induced apoptotic pathways: one is transducin-dependent and the other is transducin-independent (Hao et al., 2002). The types of gene regulatory networks that are involved or predominant in these two types of apoptosis have not been clearly established. Therefore, we aimed to delineate the signaling network in order to determine which network specifically participates in light-induced apoptosis.

\section{Materials and Methods}

\section{Animals}

All procedures involving animals were performed in accordance with the Association for Research in Vision and Ophthalmology (ARVO) Statement on the use of animals in ophthalmic and vision research. Rhodopsin kinase $\left(\mathrm{Rhok}^{-/}\right)$, arrestin $\left(\mathrm{Sag}^{-/-}\right)$, transducin $\left(\mathrm{Gna1}^{-/-}\right)$, and c-Fos $\left(\mathrm{c}-\mathrm{Fos}^{-/}\right)$knockout mice were generated as described elsewhere (Chen et al., 1999a, b; Hafezi et al., 1997; Calvert et al., 2000). Sag ${ }^{-/-}$and Gna1 $1^{-/-}$mice were crossed to each other in order to obtain arrestin/ transducin double-deficient mice $\left(\mathrm{Sag}^{-/ /} \mathrm{Gna}{ }^{-/}\right)$. All of the mice, including the wild type (WT) mice, were reared in darkness until the given experiments were performed. Wild type mice were derived from an initial cross of 129Sv and C57BL/6. The mice used in this study ranged from 6 to 8 weeks of age.

\section{Light illumination}

The mice reared in the dark were placed in aluminum foil-wrapped polycarbonate cages that were covered with stainless steel wire tops in order to protect them from uncontrolled light exposure. Fluorescent lamps provided light from an opening at the top of the cage. The mice were supplied with food and water through the bottom of the cage. Constant illumination of 2,000 lux without dilation or 6,000 lux on dilated pupils (1\% Cyclogyl, Alcon;
5\% Phenylephrine, Ciba Vision) was generated by diffused cool white fluorescent lamps and applied for various time periods ( $24 \mathrm{~h}$ for 2,000 lux or 80 $\min$ for 6,000 lux). The temperature was kept at $25^{\circ} \mathrm{C}$ during irradiation. After light exposure, the mice were either analyzed immediately or after a given period in darkness. Retinas were removed rapidly through a slit in the cornea and frozen in liquid nitrogen until total RNA was extracted using the Trizol method (Invitrogen Life Technologies). Retinas from three to four mice were pooled to make the corresponding sample.

\section{Analysis of retinal morphology}

Eyes obtained from anesthetized mice were enucleated and dissected in 1/2 Karnovsky buffer (2.5\% glutaraldehyde, $2.0 \%$ paraformaldehyde in $0.1 \mathrm{M}$ cacodylate buffer, $\mathrm{pH}$ 7.2). Eyecups were fixed overnight in $1 / 2$ Karnovsky buffer and further fixed for $2 \mathrm{~h}$ in $1 \%$ osmium tetroxide. Eyecups were embedded in epoxy resin (EPON), and 1- $\mu \mathrm{m}$ thick sections were cut along the vertical meridian at the optic nerve as described previously (Chen et al., 2006). Photomicrographs were acquired at $40 \times$ magnification using AxioVision LE Rel. 4.1. software on a Zeiss Axioskop-2 microscope (Zeiss, Göettingen, Germany).

\section{Quantification of apoptosis in the retina}

Light-induced apoptosis in the retina was quantified using a cell death detection ELISA assay kit (Roche Molecular Biochemicals) that quantifies the soluble mono- and oligo-nucleosomes released in the cell lysate as a function of apoptosis (Leist et al., 1994; Harada et al., 2000). One retina was homogenized with a $26-1 / 2$ gauge needle in $200 \mu \mathrm{l}$ of PBS with $1 \mathrm{mM}$ PMSF. The lysate was centrifuged at $15,000 \times g$ for $10 \mathrm{~min}$ at room temperature. A total of $100 \mu$ of supernatant was diluted 10 times with lysis buffer, and $20 \mu$ of diluted supernatant was used in the ELISA measurement according to the manufacturer's instructions.

\section{Microarray analysis}

First strand cDNA was synthesized using T7-oligo dT primer and SuperScript II (Invitrogen Life Technologies) with $3 \mu \mathrm{g}$ of total RNA from retinas. Second strand cDNA was synthesized with second strand buffer (Invitrogen Life Technologies), DNA polymerase I (New England Biolabs, Inc.), DNA ligase (NEB) and RNase $\mathrm{H}$ (Invitrogen Life Technologies). cDNA was extracted using phenol:chloroform:isoamyl alcohol, precipitated with ethanol, 
washed with $80 \%$ and $100 \%$ cold ethanol, and air dried. The dried pellet was then dissolved in $22 \mu \mathrm{l}$ of nuclease-free water and stored at $-20^{\circ} \mathrm{C}$. In vitro transcription was performed using the RNA Transcript Labeling Kit (Enzo Diagnostics) to produce hybridizable biotin-labeled RNA targets. The cDNA was used as a template in the presence of a mixture of unlabeled NTPs and biotinylated CTP and UTP. After in vitro transcription, CRNA was purified using an RNeasy Mini Kit (Qiagen Inc.). The fragmented cRNA, generated by incubation at $94^{\circ} \mathrm{C}$ for $35 \mathrm{~min}$, was applied to the Affymetrix GeneChip U74Av2 array (total 12,488 probe sets) and hybridized at $40^{\circ} \mathrm{C}$ for $16 \mathrm{~h}$. After hybridization, the array was washed several times and stained with streptavidin-conjugated phycoerythrin in the GeneChip Fluidics Station 400 (Affymetrix, Inc.). The arrays were scanned by the Agilent Scanner (Agilent Technologies) and analyzed using the GeneChip Analysis Suite 5.0 (Affymetrix, Inc.).

\section{Results}

\section{Morphological analysis}

Figure 1 shows retinal sections from mice after exposure to light for various time periods. No damage was detected in the wild type mice under every illumination condition tested: 6,000 lux on dilated pupils for $80 \mathrm{~min}$ (data not shown), 2,000 lux without dilation for $24 \mathrm{~h}$ (Figure 1B), and 6,000 lux on dilated pupils for 80 min and then dark adaptation for $24 \mathrm{~h}$ (Figure 1C). The knockouts that did not show any alteration in morphology on exposure to light are not shown in Figure 1. Little or no damage was discernible in the Sag ${ }^{-/}$, Gnat $1^{-/}$, $\mathrm{Sag}^{-1 /} / \mathrm{Gnat}^{-1 /}$ or $\mathrm{C}-\mathrm{Fos}^{-/}$mice after exposure to 6,000 lux for 80 min (Figure 1E, $H$ and $M$ ) and 2,000 lux for $24 \mathrm{~h}$ (Figure $1 \mathrm{~K}$ and $\mathrm{N}$ ), except for a significant degeneration in $\mathrm{Sag}^{-/}$mice exposed to 2,000 lux for $24 \mathrm{~h}$ (Figure 1I). Rod outer segments (ROS) and rod inner segments (RIS) were disorganized, the outer nuclear layer (ONL) was thinner, and pyknotic bodies were seen in Sag ${ }^{-/}$mice (mainly in inferior region) exposed to 2,000 lux for $24 \mathrm{~h}$. ROS

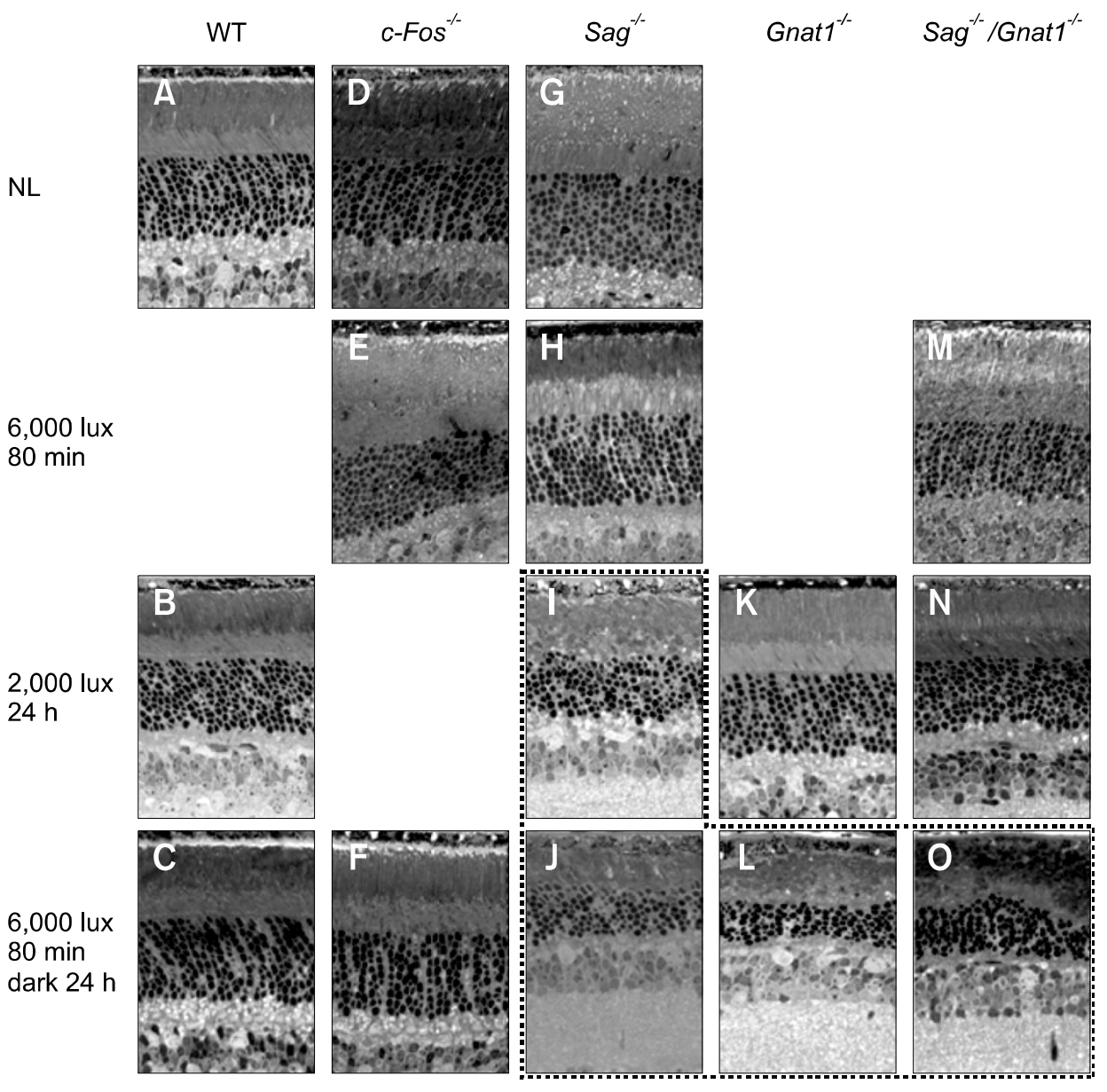

Figure 1. Retinal sections from mice after exposure to light for various time periods. The dot box indicates severe cell damage. Some pictures taken under certain conditions and at different time points where morphological changes did not occur were not included. NL, no light; WT, wild type; Sag ${ }^{-/}$, arrestin knockout; Gnat $^{--}$, transducin knockout; Sag ${ }^{-1 /}$ Gnat $^{-1 / 2}$, arrestin/transducin double knockout; c-Fos ${ }^{-/}$, c-Fos knockout. 
and RIS were disorganized, and the ONL became thin in $\mathrm{Sag}^{-/}$, Gnat $1^{-/}$or $\mathrm{Sag}^{-/} / \mathrm{Gnat}^{-/-}$mice exposed to 6,000 lux for 80 min and dark adaptation for $24 \mathrm{~h}$ (Figure $1 \mathrm{~J}, \mathrm{~L}$ and $\mathrm{O}$ ), indicating that the strong induction of apoptotic signaling by exposure to high intensity light $(6,000$ lux $)$ started before 80 min, albeit with a delayed kinetics in the knockouts. However, under the same condition $(6,000$ lux for $80 \mathrm{~min}$ and dark adaptation for $24 \mathrm{~h}$ ), the $\mathrm{c}-\mathrm{Fos}^{-/}$ mice showed a completely intact morphology (Figure 1F), while all of the Sag ${ }^{-/}$, Gnat ${ }^{-/}$and $\mathrm{Sag}^{-/} / \mathrm{Gnat}^{-/-}$mice showed severe cell damage (Figure $1 \mathrm{~J}, \mathrm{~L}$ and $\mathrm{O}$ ).

\section{Apoptosis}

We used the nucleosome release assay to measure apoptotic cell death (Leist et al., 1994; Harada et al., 2000). Whole retinas were obtained from control or irradiated mice under each condition, and damage to DNA derived from the initiation of apoptosis was gauged by measuring the number of nucleosomes released. As shown in Figure 2, nucleosome release was clearly observed in $\mathrm{Sag}^{-1-}$ after exposure to 2,000 lux for $24 \mathrm{~h}$ and $\mathrm{Sag}^{-/}$, Gnat ${ }^{-/-}$and $\mathrm{Sag}^{-/} / \mathrm{Gnat}^{-/-}$after exposure to 6,000 lux for $80 \mathrm{~min}$ and dark adaptation for $24 \mathrm{~h}$, indicating high levels of apoptosis.

\section{General gene expression patterns of regulation}

DNA microarray analysis was performed in order to identify the genes regulated by these apoptotic conditions. The numbers of genes with $\geq 2$-fold changes in expression level in various knockout mutants were counted under different conditions of light intensity (Figure 3 ). These mice were raised in a dark room and placed in bright light (2,000 lux) without pupil dilation for $24 \mathrm{~h}$ or in very bright light (6,000 lux) with pupil dilatation for $80 \mathrm{~min}$. While many up- or down-regulated genes were detected in wild type, $\mathrm{Sag}^{-/-}$or $\mathrm{Sag}^{-/ /} / \mathrm{Gnat1}^{-/-}$mice, relatively fewer differentially expressed genes were observed in Gnat1 or c-Fos KO mice as a result of light exposure (2,000 lux), suggesting that both Gnat1 and $c$-Fos are required in order to mediate light signaling or light-induced apoptotic molecular changes. In addition, it was also clear that a cascade of gene transcripts were differentially expressed as a result of the corresponding gene knockouts, such as in $\mathrm{Sag}^{-/}$.

\section{Expression levels of target genes in wild type and knockout mice}

We confirmed the expression of target genes in order to determine whether they were effectively knocked out in the corresponding knockout (Table 1). The Sag gene was effectively knocked out by $-\log _{2} 8.8$ at $0 \mathrm{~h},-\log _{2} 8.4$ at $24 \mathrm{~h}$ and $-\log _{2} 10$ in the 80 -min sample of $\mathrm{Sag}^{-/-}$retinas. Significant downregulation of Gnat1 was indicated by $-\log _{2} 9$ at $0 \mathrm{~h}$, $-\log _{2} 9.4$ at $24 \mathrm{~h},-\log _{2} 9$ in the 80 min sample.

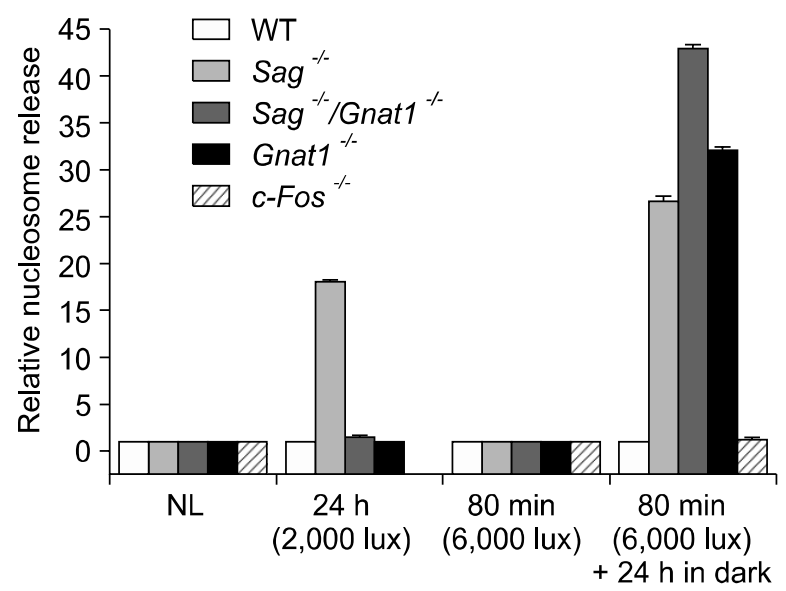

Figure 2. Nucleosome release was clearly observed in $\mathrm{Sag}^{-/}$mice exposed to 2,000 lux for $24 \mathrm{~h}$ and Sag ${ }^{-1}$, Gnat $1^{-1 /}$ and Sag ${ }^{-1 /} /$ Gnat $^{-1 /}$ exposed to 6,000 lux for $80 \mathrm{~min}$ and dark adaptation for $24 \mathrm{~h}$, indicating high levels of apoptosis. NL, no light; WT, wild type.

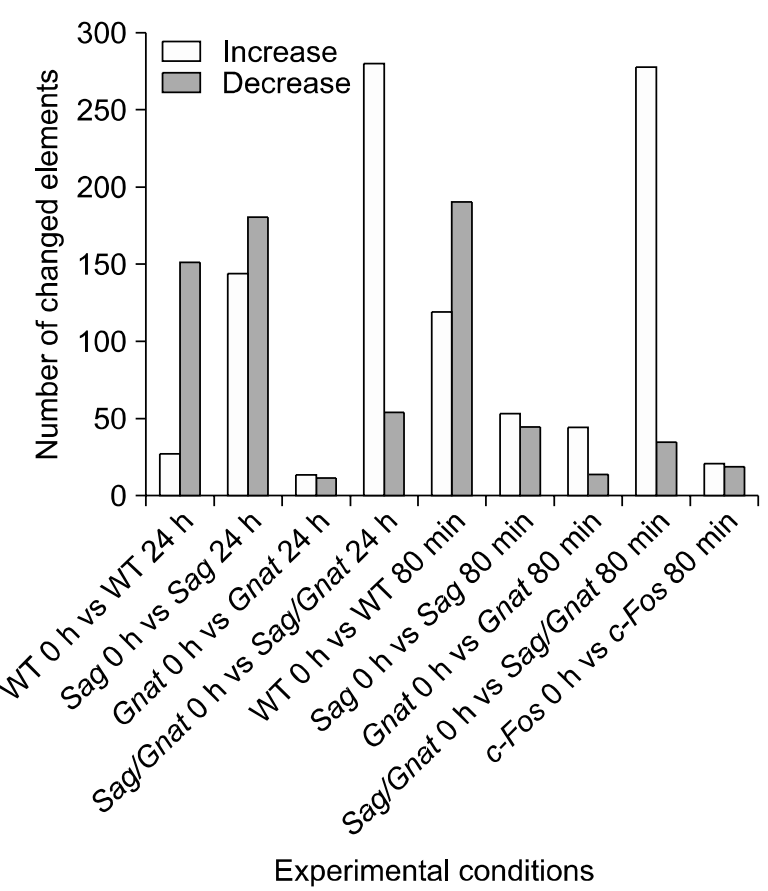

Figure 3. Number of up-regulated or down-regulated genes in $\mathrm{Sag}^{-/}$,

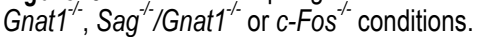



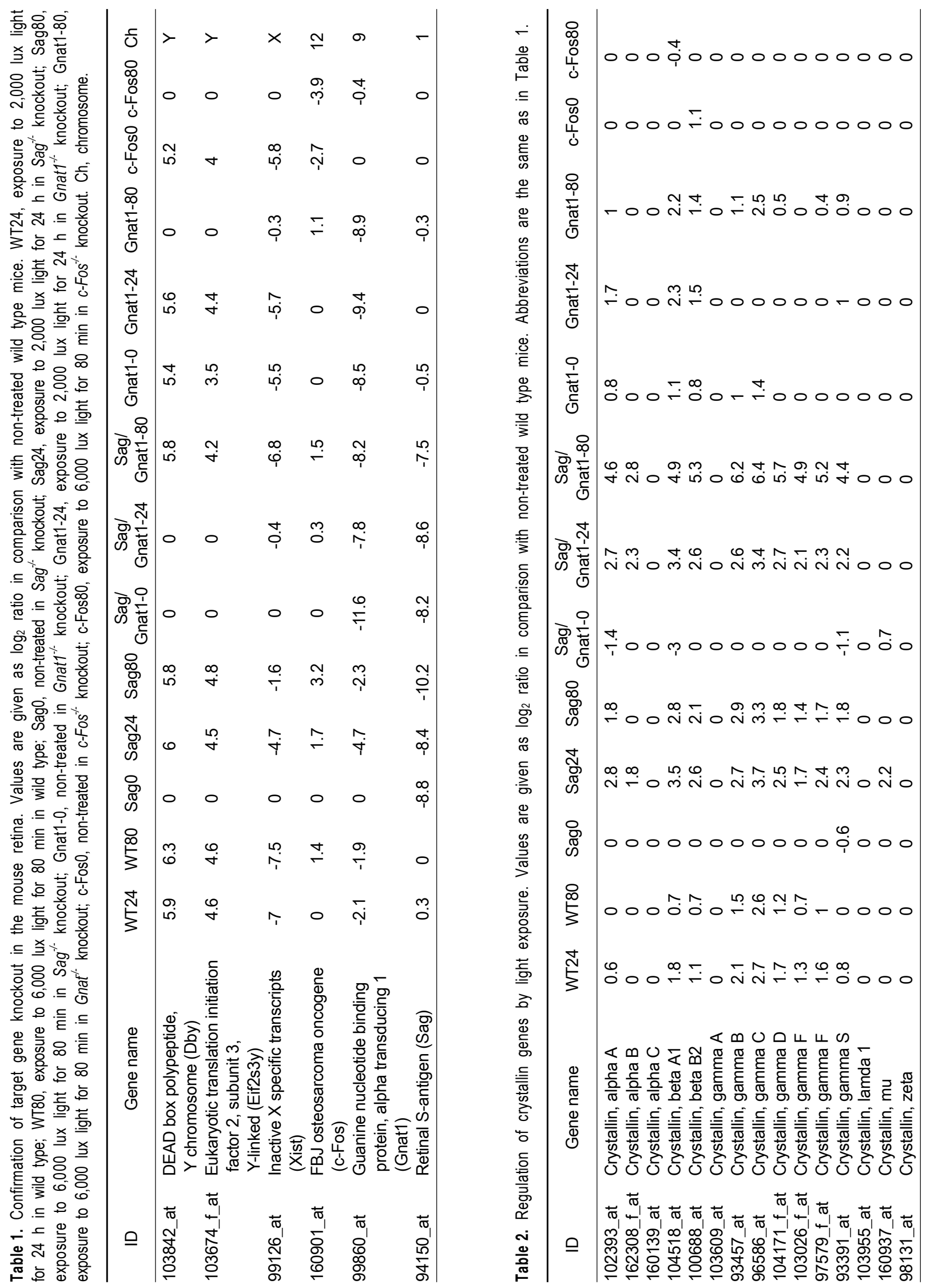


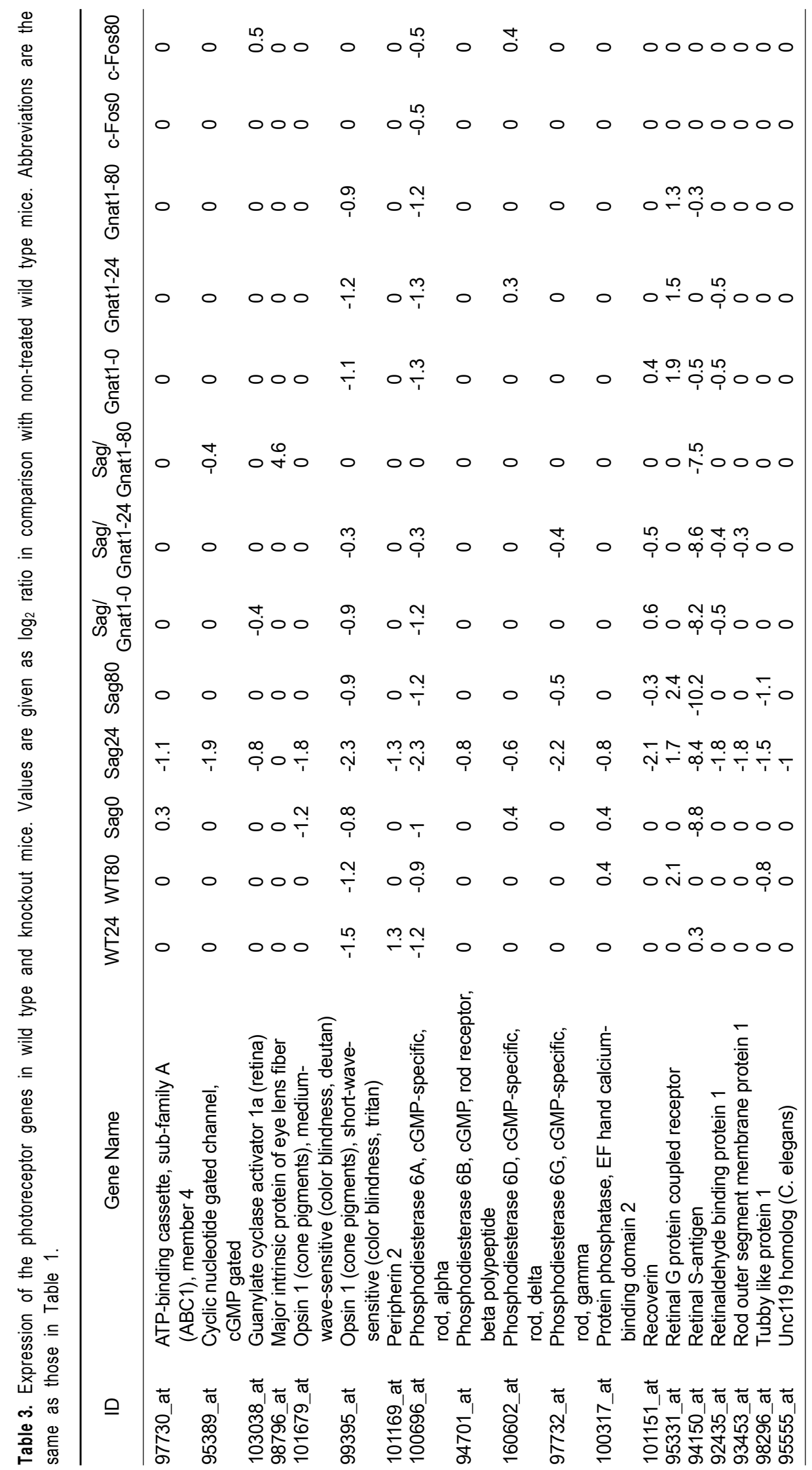




\section{A}

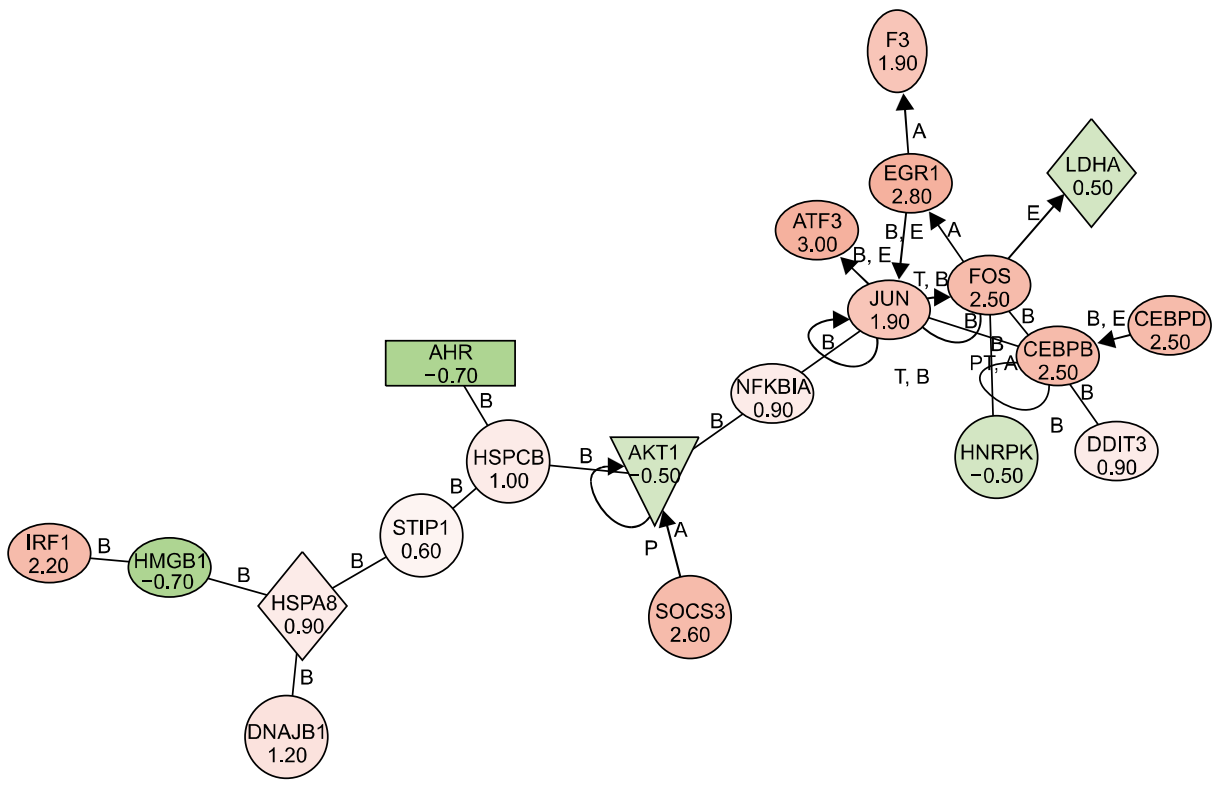

B

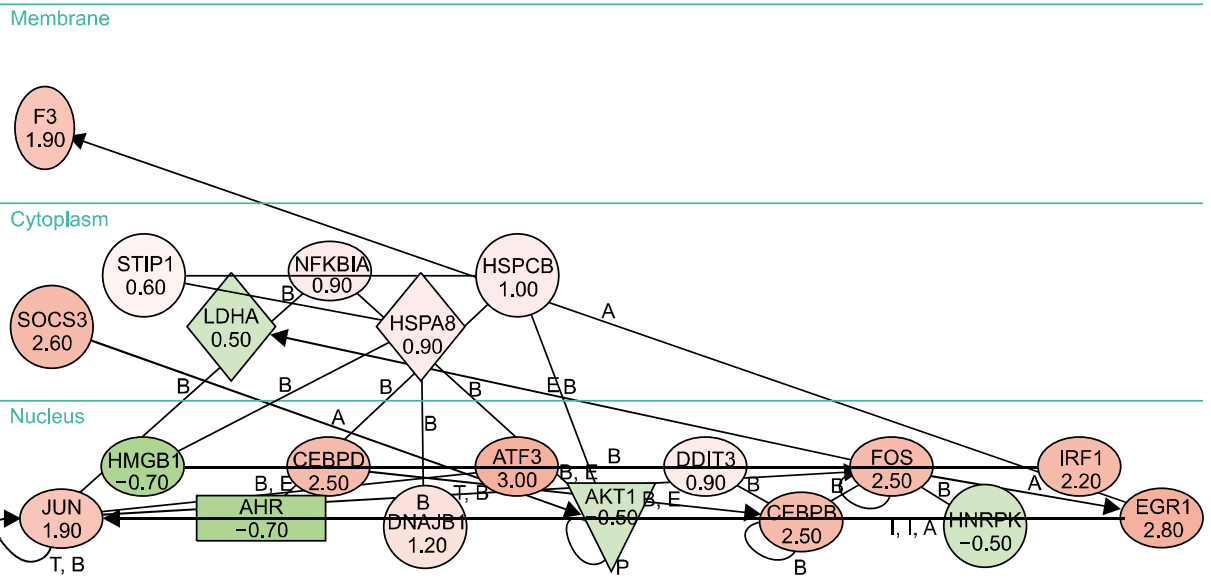

Figure 4. Graphic view of biological network 1, which was generated using the Ingenuity Pathway Analysis program. Genes with a $\log _{2}$ ratio of 0.5 or greater and -0.5 or lower were used to generate biological networks in Rhok ${ }^{-1}$ mice exposed to $1 \mathrm{~h}$ of 2,000 lux and $3 \mathrm{~h}$ of dark adaptation. Pink and red denote the up-regulated genes while dark and light green correspond to the downregulated genes. (A) Spatial layout. (B) Subcellular layout.

\section{Crystallin genes were induced only by bright light}

Intense light exposure was reported to increase crystallin content in the rat retina (Sakaguchi et al., 2003). The Coomassie blue staining intensity of crystallin 2D gel components was 2 to 3 times greater in the light-exposed retinas than in the control retinas. Neither wild type nor $\mathrm{Rhok}^{-/ /} / \mathrm{Sag}^{-1 /}$ mice exposed to low light (450 lux) showed any significant induction of crystalline gene species (alpha B \& C, beta B2, gamma A, B, C, D, \& F, lamda 1, and mu) (Krishnan et al., 2008). However, when we surveyed the expression levels at $24 \mathrm{~h}$ after continuous illumination of 2,000 lux without dilation or 80 min after illumination of 6,000 lux with dilation, the expression levels of crystallin alpha $A$, alpha $B$, beta $A 1$, beta $B 2$, gamma $B$, gamma $C$, gamma $D$, gamma $F$, and gamma $S$ were remarkably induced in both wild type and knockout mice,

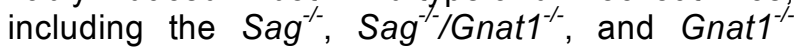
mice (Table 2). This finding supports the fact that only bright light can induce crystallin genes. Recent studies have shown that an increase in the chaperone ability of alpha-crystallin at higher temperatures can protect target proteins from aggregating and precipitating (Ecroyd et al., 2007; Rekas et al., 2007). There are two main crystallin gene families: the alpha-crystallins and beta/ gamma-crystallins. The beta/gamma-crystallin family 


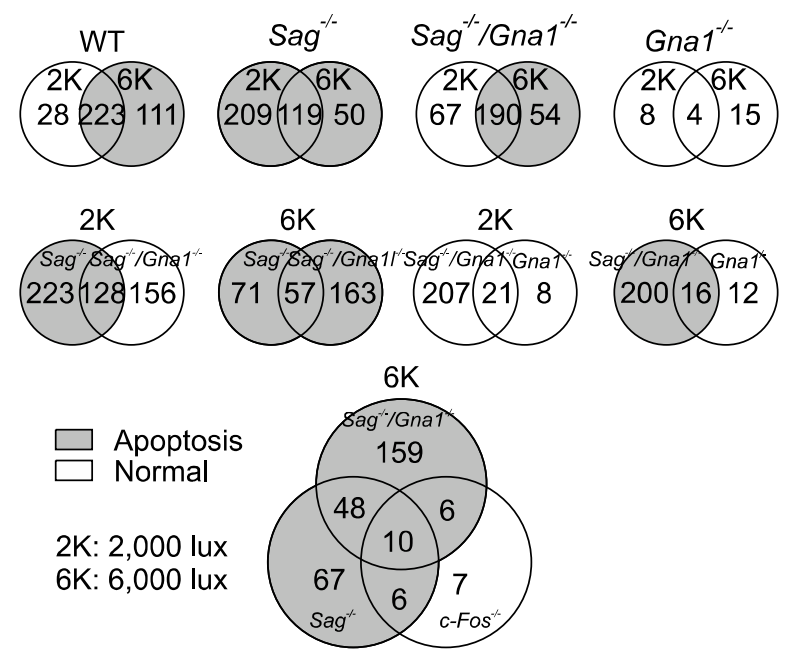

Figure 5. Venn diagrams of regulated ( $\geq 2$ fold) genes in $\mathrm{Sag}^{-/}$,

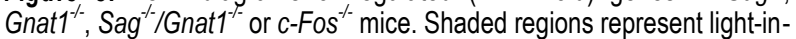
duced apoptotic conditions while white regions correspond to normal conditions.

has been suggested to affect lens development (Andley, 2007). The increased levels of beta/ gamma-crystallins in bright light conditions may indicate a new function of beta/gamma-crystallins that is related to stress.

\section{Photoreceptor genes were affected by different mechanisms}

As shown in Table 3, the expression of photoreceptor genes was significantly down-regulated in $\mathrm{Sag}^{-/}$ mice $(2,000$ lux without pupil dilation for $24 \mathrm{~h})$, indicating that the progression of apoptosis (Figure 2 ) induced remarkable structural changes (Figure 1I). The surprising observation was that this downregulation of photoreceptor signaling genes, presumably due to photoreceptor cell damage, did not occur in $\mathrm{Sag}^{-/ /} / \mathrm{Gnat1}^{-/}$mice under the same condition $(2,000$ lux without dilation for $24 \mathrm{~h})$ (Figure $1 \mathrm{~N}$ and 2). Bright light (5,000 lux for $20 \mathrm{~min}$ or 1,700 lux for 7 days) has been reported to trigger apoptosis of photoreceptor cells through the induction of AP-1. On the other hand, photoreceptor cell apoptosis in $\mathrm{Rhok}^{-/}$or Sag ${ }^{-/}$mice exposed to low intensity light is dependent on transducin and does not require AP-1 (Hao et al., 2002). When exposed to 2,000 lux for $24 \mathrm{~h}$, the molecular responses of $\mathrm{Sag}^{-/} / \mathrm{Gnat}^{-/ /}$mice to photoreceptor genes were quite different from those of $\mathrm{Sag}^{-/-}$mice but similar to those of wild type or Gnat $1^{-/}$mice (Figure 1 and 2), indicating that an additional knockout of the Gnat1 gene causes a delayed response and/or ramification of signaling pathways in $\mathrm{Sag}^{-/ /} / \mathrm{Gnat}^{-1 /-}$ mice. Therefore, it is more likely that apoptotic signaling requires the involvement of transducin under this condition.

\section{Signal transduction networks}

In light-induced retinal degeneration, as well as in inherited retinal degeneration, apoptosis is the "common pathway" of photoreceptor cell death. Therefore, we studied the signaling of stress-induced photoreceptor apoptosis mechanisms by utilizing the pathway analysis program (Ingenuity). We used a subset of regulated genes ( $>0.5$ or $<-0.5$ in $\log _{2}$ ratio) under specific conditions in order to obtain insights into the mechanisms of cell death and signaling. In $1 \mathrm{~h}$ illumination of 2,000 lux in $R_{h o k^{-/}}$mice, we observed 2 major networks (scored equal to or above 7). The 1st network constitutes EGR1, Fos, and NR4A1, which seem to be the major proteins of apoptosis. It also includes BDNF, CEBP delta, CTSB (Cathepsin B), and DUSP1 (dual specificity phosphatase 1 ), which function in the developmental process. As the amount of dark adaptation time increases, the number of major networks increases. Six major networks (scored equal to or above 7) were observed after $3 \mathrm{~h}$ of dark adaptation followed by an hour of light illumination. After $3 \mathrm{~h}$ of dark adaptation the first network, which consisted of AHR, AKT1, ATF3, CEBPbeta, CEBPdelta, DDIT3, DNAJB1, EGR1, F3, Fos, HMGB1, HNRPK, HSPA8, HSPCB, IRF1, Jun, LDHA, NFKBIA, SOCS3, and STIP1, obtained the highest score of 35 (Figure 4). In addition, all 20 members of this particular network were being regulated in that condition. Therefore, it is most likely that this network plays a very critical role in the mutant, and its role may be in apoptosis.

\section{Lessons from knockout experiments}

The Venn diagrams (Figure 5) show the numbers of regulated genes (more than 2 fold) between conditions. Several conclusions can be derived from these Venn diagrams. First, there were 362 gene elements in wild type retinas regulated by light exposure itself. Second, a different set of genes was regulated by exposure to 2,000 lux or 6,000 lux of light in all conditions tested. Third, the transducin knockout seems to block phototransduction signals triggered by exposure to both 2,000 lux and 6,000 lux of light. Fourth, transducin appears to play an important role in apoptotic signaling in arrestin knockout $\left(\mathrm{Sag}^{-/ /}\right)$mice exposed to 2,000 lux of light but not in those exposed to 6,000 lux of light. Fifth, the $c$-Fos knockout appears to block photo signal transduction under 6,000 lux of light. 


\section{Discussion}

Retinal degeneration has long been a subject of intense investigation due to its important clinical applications. In the present study, we examined the effects of constant light-induced phototoxicity and signaling networks. We performed gene expression analyses to facilitate the identification of regulated genes in the retinas of different knockout mice under different conditions. When the number of regulated genes was measured in each knockout or under each light condition, significant molecular changes, which eventually induced retinal cell degeneration, were observed in $\mathrm{Sag}^{-/}$, Gnat1 ${ }^{-/-}$or $\mathrm{Sag}^{-/} / \mathrm{Gnat}^{-/-}$knockouts (Figure $1 \mathrm{I}, \mathrm{J}, \mathrm{L}, \mathrm{O}$ and 2). These results suggest that such knockouts may be at greater risk of light-induced damage.

Intense light exposure was reported to change the crystallin content in the retina (Andley, 2007; Ecroyd et al., 2007; Rekas et al., 2007). Alphacrystallin has antioxidant as well as antiapoptotic functions, and it can protect enzymes and other crystallins against both chemically- and thermallyinduced inactivation or aggregation, which may play an important role in maintaining the transparency of the lens (Manzanares et al., 2001; Xi et al., 2003; Liu et al., 2004; Mao et al., 2004). When we surveyed the expression levels $24 \mathrm{~h}$ after continuous illumination of 2,000 lux without dilation or 80 min after illumination of 6,000 lux with dilation, the expression levels of crystalline alpha $A$, alpha $B$, beta $A 1$, beta $B 2$, gamma $B$, gamma $C$, gamma $D$, gamma $F$ and gamma $S$ were remarkably increased. As a chaperone and heat shock protein, alpha-crystallins can serve as a regulator of protein conformation and as stress sensors. The ability of crystallins to interact with free radicals and remove hypochlorous acid could potentially contribute to the maintenance of the lens functionally. Many chaperones play a role in regulating cell proliferation and apoptosis (Mosser and Morimoto, 2004).

When we further explored the fate of the photoreceptor genes, we found that their expression levels were significantly down-regulated in $\mathrm{Sag}^{-/-}$ mice, representing remarkable structural changes due to the progression of apoptosis (Figure 1I). A surprising observation was that this down-regulation of photoreceptor signaling genes, presumably due to photoreceptor cell damage, did not occur in $\mathrm{Sag}^{-/} / \mathrm{Gnat1}^{-/-}$mice under the same condition (Figure $1 \mathrm{~N}$ ). The deletion of transducin seems to block the light-related apoptotic signal. This result supports that photoreceptor cell apoptosis in Rhok ${ }^{-/}$ or $\mathrm{Sag}^{-/-}$mice exposed to low light is dependent on transducin and does not require c-Fos (Hao et al.,
2002).

Relatively fewer genes were differentially expressed in $\mathrm{Gnat1}^{-/-}$and $\mathrm{C}-\mathrm{Fos}^{-/-}$mice exposed to light (Figure 3). c-Fos is a component of the dimeric transcription factor AP-1, and it must combine with Jun proteins to form a functional unit (Angel and Karin, 1991). Ablation of c-Fos protects photoreceptors from light-induced damage (Hafezi et al., 1997). In most of the conditions tested, including the wild type, c-Fos was significantly up-regulated by exposure to bright light (except in c-Fos knockouts) (Table 1), confirming the important role of AP-1 in light-induced stress.

In the present study, we found out that different cascades of gene components were induced or inhibited as a result of corresponding gene knockouts under specific light conditions. For example, the expression of the crystalline gene was increased in the retina by exposure to bright light. The deletion of transducin seems to block light-related apoptotic signaling in Sag ${ }^{-/}$mice. c-Fos has been significantly up-regulated as a light stress signal, while knockout of the c-Fos gene appears to block bright lightinduced retinal degeneration. These results, therefore, provide an impetus to construct retinal photoreceptor signaling pathways.

\section{Acknowledgement}

This work was supported by the Korea Research Foundation Grant funded by the Korean Government (MOEHRD) (KRF-2006-311-C00482), and it was also partly supported by a Korea Science and Engineering Foundation (KOSEF) grant funded by the Korean government (MOST) (R01-2007-000-20533-0).

\section{References}

Andley UP. Crystallins in the eye: Function and pathology. Prog Retin Eye Res 2007;26:78-98

Angel P, Karin M. The role of Jun, Fos and the AP-1 complex in cell-proliferation and transformation. Biochim Biophys Acta 1991;1072:129-57

Calvert PD, Krasnoperova NV, Lyubarsky AL, Isayama T, Nicolo M, Kosaras B, Wong G, Gannon KS, Margolskee RF, Sidman RL, Pugh EN, Jr, Makino CL, Lem J. Phototransduction in transgenic mice after targeted deletion of the rod transducin alpha -subunit. Proc Natl Acad Sci USA 2000;97: 13913-8

Chang GQ, Hao Y, Wong F. Apoptosis: final common pathway of photoreceptor death in rd, rds, and rhodopsin mutant mice. Neuron 1993;11:595-605

Chen CK, Burns ME, Spencer M, Niemi GA, Chen J, Hurley JB, Baylor DA, Simon MI. Abnormal photoresponses and light-induced apoptosis in rods lacking rhodopsin kinase. 


\section{Proc Natl Acad Sci USA 1999a;96:3718-22}

Chen J, Simon MI, Matthes MT, Yasumura D, LaVail MM. Increased susceptibility to light damage in an arrestin knockout mouse model of Oguchi disease (stationary night blindness). Invest Opthalmol Vis Sci 1999b;40:2978-82

Chen J, Shi G, Concepcion FA, Xie G, Oprian D, Chen J. Stable rhodopsin/arrestin complex leads to retinal degeneration in a transgenic mouse model of autosomal dominant retinitis pigmentosa. J Neurosci 2006;26:11929-37

Choi S, Hao W, Chen CK, Simon MI. Gene expression profiles of light-induced apoptosis in arrestin/rhodopsin kinase-deficient mouse retinas. Proc Natl Acad Sci USA 2001;98:13096-101

Dolph PJ. Arrestin: roles in the life and death of retinal neurons. Neuroscientist 2002;8:347-55

Ecroyd H, Meehan S, Horwitz J, Aquilina JA, Benesch JL, Robinson CV, Macphee CE, Carver JA. Mimicking phosphorylation of alphaB-crystallin affects its chaperone activity. Biochem J 2007;401:129-41

Hafezi F, Steinbach JP, Marti A, Munz K, Wang ZQ, Wagner $E F$, Aguzzi A, Reme CE. The absence of $c$-fos prevents light-induced apoptotic cell death of photoreceptors in retinal degeneration in vivo. Nat Med 1997;3:346-9

Hao W, Wenzel A, Obin MS, Chen CK, Brill E, Krasnoperova NV, Eversole-Cire P, Kleyner Y, Taylor A, Simon MI, Grimm C, Reme CE, Lem J. Evidence for two apoptotic pathways in light-induced retinal degeneration. Nat Genet 2002;32: 254-60

Harada T, Harada C, Nakayama N, Okuyama S, Yoshida K, Kohsaka S, Matsuda H, Wada K. Modification of glialneuronal cell interactions prevents photoreceptor apoptosis during light-induced retinal degeneration. Neuron 2000;26: 533-41

Krishnan J, Lee G, Han SU, Choi S. Characterization of phototransduction gene knockouts revealed important signaling networks in the light-induced retinal degeneration. J Biomed Biotechnol 2008;2008:327468

Leist M, Gantner F, Bohlinger I, Germann PG, Tiegs G,
Wendel A. Murine hepatocyte apoptosis induced in vitro and in vivo by TNF-alpha requires transcriptional arrest. $J$ Immunol 1994;153:1778-88

Liu JP, Schlosser R, Ma WY, Dong Z, Feng H, Lui L, Huang $X Q$, Liu Y, Li DW. Human alphaA- and alphaB-crystallins prevent UVA-induced apoptosis through regulation of PKCalpha, RAF/MEK/ERK and AKT signaling pathways. Exp Eye Res 2004;79:393-403

Makino CL, Wen XH, Lem J. Piecing together the timetable for visual transduction with transgenic animals. Curr Opin Neurobiol 2003;13:404-12

Manzanares D, Bauby C, de la Pena R, Garcia JC, Sanchez R, Martinez S, Romay CH, Lopez-Reconde JL, Pino E, Lissi EA. Antioxidant properties of alpha-crystallin. J Protein Chem 2001;20:181-9

Mao YW, Liu JP, Xiang H, Li DW. Human alphaA- and alphaB-crystallins bind to $\mathrm{Bax}$ and $\mathrm{Bcl}-\mathrm{X}(\mathrm{S})$ to sequester their translocation during staurosporine-induced apoptosis. Cell Death Differ 2004;11:512-26

Miller WE, Lefkowitz RJ. Arrestins as signaling molecules involved in apoptotic pathways: a real eye opener. Sci STKE 2001;2001:PE1

Mosser DD, Morimoto RI. Molecular chaperones and the stress of oncogenesis. Oncogene 2004;23:2907-18

Portera-Cailliau C, Sung CH, Nathans J, Adler R. Apoptotic photoreceptor cell death in mouse models of retinitis pigmentosa. Proc Natl Acad Sci USA 1994;91:974-8

Rekas A, Jankova L, Thorn DC, Cappai R, Carver JA. Monitoring the prevention of amyloid fibril formation by alpha-crystallin. Temperature dependence and the nature of the aggregating species. FEBS J 2007;274: 6290-4

Sakaguchi H, Miyagi M, Darrow RM, Crabb JS, Hollyfield JG, Organisciak DT, Crabb JW. Intense light exposure changes the crystallin content in retina. Exp Eye Res 2003;76:131-3

Xi J, Farjo R, Yoshida S, Kern TS, Swaroop A, Andley UP. A comprehensive analysis of the expression of crystallins in mouse retina. Mol Vis 2003;9:410-9 